\title{
Idade para expedição de mudas clonais de eucalipto em função de variáveis morfológicas não destrutivas
}

\author{
Glauciana da Mata Ataíde ${ }^{1 *}$, Renato Vinicius Oliveira Castro ${ }^{1}$, Bruna Anair Souto Dias ${ }^{2}$, Ana Flávia Neves Mendes \\ Castro ${ }^{1}$
}

${ }^{1}$ Universidade Federal de São João Del-Rei, Campus Sete Lagoas, Rodovia MG-424, km47, Sete Lagoas - MG, 35701-970

2 Universidade Federal do Piauí, Campus Universitário Professora Cinobelina Elvas, BR 135, km 3, Bom Jesus - PI, 64900-000

*Autor para correspondência: glauciana@ufsj.edu.br

Recebido: Maio 2019/ Aceito: Novembro 2019 / Publicado: Dezembro 2019

\section{Resumo}

A idade de expedição das mudas de eucalipto do viveiro é baseada, geralmente, em indicadores visuais, associados às variáveis altura e diâmetro. Este trabalho objetivou avaliar estas variáveis (não destrutivas) na determinação da idade de expedição de mudas de eucalipto e indicar critérios para a definição da idade adequada para expedição. Foram avaliadas mudas de quatro clones de Eucalyptus grandis x E. urophylla. Foram medidas as variáveis altura (H), diâmetro do colo (DC) e relação altura/diâmetro do colo (H/DC) aos 15, 40 e 55 dias após o estaqueamento (DAE). Ajustou-se o modelo Logístico para estudar o comportamento das variáveis, sendo utilizado um modelo de decisão multicritério para definir a idade de expedição, ao comparar qual se ajusta mais eficientemente às condições: C1: Incremento Médio Diário (IMD) se iguala ao Incremento Corrente Diário (ICD) para a altura; $C 2$ : DC $\geq 2,0$ mm; C3: H/D com valores entre 5,4 e 8,1. Após análise dos critérios e do comportamento dos clones em função destes, observou-se que a utilização dos critérios definidos para altura e diâmetro possibilitou a definição da idade de expedição das mudas. No entanto, a relação H/D não foi eficiente para esta definição. Conclui-se que os critérios $\mathrm{C} 1 \mathrm{e}$ C2 definidos para as variáveis altura e diâmetro foram eficientes para determinação da idade de expedição das mudas e, com base nestes, foi definida a idade de expedição entre 50 e 59 DAE para os clones de Eucalyptus grandis x $E$. urophylla estudados.

Palavras-chave: Altura, Diâmetro, Modelagem, Viveiros florestais.

\begin{abstract}
The age of expedition of eucalyptus seedlings from the nursery is based generally on visual indicators, associated with the variables height and diameter. This study aimed to evaluate the non-destructive morphological variables in the determination of age of expedition of eucaly ptus seedlings of four clones of Eucalyptus grandis x E. urophylla. Were measured the variables height $(\mathrm{H})$, diameter (DC) and ratio of height / diameter (H/DC) at 15, 40 and 55 days after cutting (DAC). The logistic model was set to study the behavior of the variables. Were used a model of multi-criteria decision to define the age of expedition, to compare which fits more efficiently conditions: $\mathrm{C} 1$ : Increased average daily (IMD) equals the current increment daily (ICD) for height; C2: DC $\geq 2.0 \mathrm{~mm} ; \mathrm{C} 3$ : $\mathrm{H} / \mathrm{D}$ values between 5.4 and 8.1 . The use of nondestructive variables allowed the definition of the age of expedition of seedlings, but the increments in diameter increase the time of seedling on nursery. Were concluded that the morphological variables used were efficient for determining the age of expedition of seedlings and, based on these, the age of expedition was between 50 and 59 DAC for clones of Eucalyptus grandis $\mathrm{x}$ E. urophylla studied.
\end{abstract}

Keywords: Height, Diameter, Modeling, Forest nurseries.

\section{Introdução}

Segundo IBÁ (2018), a área total de árvores plantadas no Brasil totalizou 7,84 milhões de hectares em 2016 , apresentando um crescimento de $0,5 \%$ em relação ao ano de 2015, devido principalmente ao aumento das áreas com espécies do gênero Eucalyptus. O eucalipto é a espécie de maior representatividade no cenário de florestas plantadas brasileiras, sendo seu cultivo um dos mais avançados, produtivos e competitivos do mundo (Souza-Silva e Zanetti 2007; Souza 2019)

A demanda crescente por plantios de eucalipto tem exigido um aumento na qualidade das mudas produzidas (Ataíde et al. 2010; Queiroz et al. 2018). A utilização de mudas com padrão adequado contribui positivamente para o êxito do povoamento florestal, através do aumento nas taxas de sobrevivência e crescimento inicial em campo (Silveira et al. 2004; Melo 2017; Tavanti et al. 2018).

A expedição de mudas de qualidade contribuirá para a redução de custos com o replantio, e o crescimento mais acelerado poderá contribuir para que superem a competição com as plantas daninhas mais rapidamente, promovendo a diminuição de gastos com tratos culturais (Figueiredo et al. 2011). No entanto, a idade ótima de expedição das mudas para o plantio é baseada, geralmente, em valores empíricos (Mafia et al 2005), considerando-se as variáveis morfológicas e fisiológicas presentes no momento da expedição.

Para avaliação do padrão de qualidade das mudas, as variáveis morfológicas não destrutivas têm sido extensivamente utilizadas (Carneiro 1995; Gomes et al. 2002; Costa e Streck 2018; Gonzaga et al. 2018; Fernandes et al. 2019), destacando-se como principais medições a altura e o diâmetro das mudas. Sua facilidade de avaliação, aliada à melhor compreensão por parte dos viveiristas, possibilita a manutenção das mudas no viveiro para atender à demanda do mercado, sem ser necessária a destruição das mudas (Gomes et al., 2002).

O desenvolvimento de metodologias de análise de dados que possibilitem a definição do momento ótimo de expedição das mudas é fundamental. Tais métodos objetivam definir, com base em modelos matemáticos, quando ocorrerá a expedição de mudas com melhor padrão de crescimento e desenvolvimento, de modo que não sigam para o campo mudas ainda prematuras e, por outro lado, não seja retardada a saída de mudas que já estão desenvolvidas.

Diante do exposto, o presente trabalho teve como objetivo avaliar a eficiência das variáveis morfológicas não destrutivas altura e diâmetro serem utilizados como base para a expedição das mudas clonais de eucalipto do viveiro e indicar critérios para a definição do momento ótimo de expedição das mudas.

\section{Material e métodos}

$\mathrm{O}$ presente trabalho foi conduzido em um viveiro de produção de mudas de eucalipto situado na cidade de Belo 
Oriente, região centro-leste do estado de Minas Gerais. As mudas de eucalipto foram provenientes do minijardim clonal da empresa, obtidas pelo enraizamento de miniestacas oriundas de plantas propagadas pelo método da miniestaquia, conforme a técnica descrita por Xavier et al. (2013).

Foram avaliados quatro clones de Eucalyptus grandis x $E$. urophylla. Para cada clone, as miniestacas, com 4 a $6 \mathrm{~cm}$ de tamanho foram estaqueadas em tubetes de plástico de $55 \mathrm{~cm}^{3}$, contendo substrato constituído de partes iguais de vermiculita de granulometria média e casca de arroz carbonizada, acrescido dos macro e micronutrientes: $8,0 \mathrm{~kg} \mathrm{~m}^{-3}$ de superfosfato simples; $0,695 \mathrm{~kg} \mathrm{~m}^{-3}$ de sulfato de amônio; $0,208 \mathrm{~kg} \mathrm{~m}^{-3}$ de cloreto de potássio; $0,014 \mathrm{~kg} \mathrm{~m}^{-3}$ de sulfato de zinco; $0,014 \mathrm{~kg} \mathrm{~m}^{-3}$ de sulfato de cobre; $0,014 \mathrm{~kg} \mathrm{~m}^{-3}$ de sulfato de manganês; e $0,028 \mathrm{~kg} \mathrm{~m}^{-3}$ de ácido bórico.

As miniestacas foram colocadas para enraizamento em casa de vegetação com temperatura e umidade controladas por 20 dias, seguindo, posteriormente para a casa de sombra, com sombrite de $50 \%$, onde permaneceram por cinco dias. As mudas recém-tiradas da casa de sombra foram medidas e transferidas para canteiros de crescimento, coberto por um teto translúcido de plástico retrátil.

A partir da saída da casa de sombra, foram realizadas mais duas avaliações nas mudas: com intervalo de 15 dias, aos 40 e 55 dias após o estaqueamento (DAE). Em cada avaliação, foram medidos a altura $(\mathrm{H})$, o diâmetro do colo $(\mathrm{D})$ e a relação entre a altura da parte aérea e o diâmetro do colo (H/D) das mudas de eucalipto dos clones estudados. A altura foi medida com uma régua milimetrada posicionada em nível do substrato até o meristema apical da muda e o diâmetro foi mensurado com paquímetro digital. A H/D foi a divisão das variáveis citadas.

Para estimar a idade ótima de expedição das mudas utilizou-se um modelo de decisão multicritério, ao combinar a associação de técnicas de modelagem e analíticas, considerando-se: Critério1: Incremento Médio Diário (IMD) se iguala ao Incremento Corrente Diário (ICD) para a variável altura da muda, de acordo com metodologia proposta por Mafia et al. (2005); Critério2: $\mathrm{D} \geq 2,3 \mathrm{~mm}$, segundo proposto por Gomes et al. (2002) para mudas de eucalipto; Critério3: H/D na faixa de valores entre 5,4 e 8,1 (Carneiro 1995).

Para estudar o comportamento das variáveis H, D e H/D; foram testados modelos sigmoidais, sendo selecionado o modelo Logístico, definido por $Y=\frac{\alpha}{1+\beta \cdot e^{\left(-\gamma_{2} . I\right)}}+\varepsilon$, em que $\mathrm{Y}=$ variável sob análise $(\mathrm{H}, \mathrm{D}$ e H/D); I= idade, em Tabela 1. Modelos, estimativas dos parâmetros e coeficiente de correlação para as variáveis H, D e H/D por tratamento em função da idade para mudas clonais de eucalipto.

\begin{tabular}{|c|c|c|c|c|c|}
\hline \multirow{2}{*}{ Modelo* } & \multirow{2}{*}{ Parâmetro } & \multicolumn{4}{|c|}{ Tratamento } \\
\hline & & Clone $1^{*}$ & Clone 2 & Clone 3 & Clone 4 \\
\hline \multirow{4}{*}{$H=\frac{\alpha}{1+\beta \cdot e^{(-\gamma . I)}}$} & $\alpha$ & 21,2807 & 20,2038 & 23,6512 & 23,4978 \\
\hline & $\beta$ & 8,2235 & 7,3034 & 10,8257 & 6,7594 \\
\hline & $\gamma$ & 0,0910 & 0,0876 & 0,0815 & 0,0691 \\
\hline & $(R \hat{y} y)$ & 0,83 & 0,87 & 0,89 & 0,89 \\
\hline \multirow{5}{*}{$D=\frac{\alpha}{1+\beta \cdot e^{(-\gamma \cdot I)}}$} & $\alpha$ & 2,4675 & 2,4897 & 2,3796 & 2,4729 \\
\hline & $\beta$ & 2,9692 & 2,4436 & 9,1684 & 2,5515 \\
\hline & $\gamma$ & 0,0927 & 0,0604 & 0,1186 & 0,0641 \\
\hline & $(R \hat{y} y)$ & 0,71 & 0,80 & 0,76 & 0,88 \\
\hline & $\alpha$ & 8,7741 & 9,4015 & 12,4922 & 9,9036 \\
\hline
\end{tabular}

dias; $\alpha=$ ponto assintótico superior; $\beta=$ ponto de inflexão e $\gamma=$ constante de integração; $\varepsilon=$ erro aleatório. Os parâmetros utilizados para qualificar o modelo Logístico foram o coeficiente de correlação (R) e o erro padrão da estimativa. Além disso, este modelo foi selecionado por ser coerente biologicamente com o crescimento de mudas florestais (Mafia et al., 2005).

A partir da análise dos dados de regressão das variáveis D e H / D, foi definida a data recomendada para a expedição das mudas de eucalipto, de acordo com os critérios estabelecidos. Para a variável $\mathrm{H}$, foram calculados $\mathrm{o}$ incremento corrente diário (ICD) e o incremento médio diário (IMD) para os quatro clones, a fim de obter os valores para o Critério1, empregando-se a função $(d Y / d T)=\gamma x Y(\alpha-Y) / \alpha$.

$\mathrm{O}$ delineamento utilizado foi o inteiramente casualizado com 20 repetições para cada clone e tempo de avaliação, sendo cada muda representativa de uma repetição. Os dados apresentaram distribuição normal $(p>0,05)$ pelo teste Shapiro-Wilk. As análises foram realizadas nos programas Statistica 7.0 (Statsoft 2017) e Excel 2010.

\section{Resultados e discussão}

De acordo com o ajuste do modelo logístico para os quatro clones (Tabela 1), as estimativas dos parâmetros e dos coeficientes para as variáveis testadas apresentaram-se significativas ao nível de $1 \%$ de probabilidade. Analisandose os coeficientes encontrados, observa-se que $\alpha$ (o ponto assintótico superior - que é o valor de y quando $\mathrm{x}$ tende ao infinito, ou seja, o valor máximo de y) variou de 20,2 a 23,5 $\mathrm{cm}$ de altura; 2,4 a $2,5 \mathrm{~mm}$ de diâmetro do coleto e 8,8 a 12,5 para a relação $H / D$. O coeficiente $\beta$, que corresponde ao ponto de inflexão do modelo, ou seja, o valor de y quando se obtém as maiores taxas de crescimento, variou de 7,3 a 10,9 cm de altura, 2,4 a 9,2 mm de diâmetro do coleto e 1,7 a 3,0 de relação $H / D$.

O coeficiente de correlação de Pearson entre os valores de y estimados e observados apresentou valores satisfatórios de ajustes para as variáveis selecionadas, principalmente para as variáveis $H$ e $D$, nas quais variou entre 0,71 e 0,89 . Para a variável $H / D$ os valores da correlação de Pearson variaram de 0,48 a 0,75 para os quatro clones estudados. Considerando os ajustes obtidos, os três modelos podem ser utilizados para a estimativa da altura, diâmetro e relação altura/diâmetro em função da idade das mudas de eucalipto. 
A taíde et al.

\begin{tabular}{clllll}
$\alpha$ & $\beta$ & 2,7695 & 3,0349 & 2,3094 & 1,7257 \\
$H=\frac{\alpha}{1+\beta \cdot e^{(-\gamma . I)}}$ & $\gamma$ & 0,0732 & 0,1070 & 0,0368 & 0,0493 \\
& $(R \hat{y} y)$ & 0,64 & 0,48 & 0,75 & 0,68 \\
\hline
\end{tabular}

*Em que $\mathrm{H}=$ altura das mudas; $\mathrm{D}=$ diâmetro do coleto das mudas; $\mathrm{H} / \mathrm{D}=$ relação altura/diâmetro das mudas; Y variável sob análise $(H, D$ e H/D); I= idade, em dias; $\alpha=$ ponto assintótico superior; $\beta=$ ponto de inflexão; $\mathrm{B} \gamma=$ constante de integração, sem interpretação biológica; $\varepsilon$ erro aleatório; $(R \hat{y} y)=$ coeficiente de correlação

Os quatro clones avaliados apresentaram comportamentos similares em relação à altura (Figura 2), com tendência de crescimento sigmoidal ao longo do período de permanência no viveiro. Inicialmente, as mudas priorizam o desenvolvimento em altura como forma de maximizar a interceptação da radiação solar, visando a maiores incrementos nas taxas fotossintéticas (Gibert et al. 2016; Guo et al. 2016). Pires et al. (2019) ressaltam que o acúmulo de fotoassimilados inicialmente na parte aérea das mudas de eucalipto resultam em incrementos em altura, e posteriormente estes fotossintatos são translocados vara as raízes, as quais funcionam como um dreno para os carboidratos produzidos pelas folhas.

Por tratar-se de uma variável de fácil medição, a altura exerce importante papel na avaliação da sobrevivência e desenvolvimento das mudas nos primeiros anos após o plantio (Carneiro 1995), sendo bastante difundida na avaliação da resposta das mudas de eucalipto às variações nos fatores de produção em viveiro.

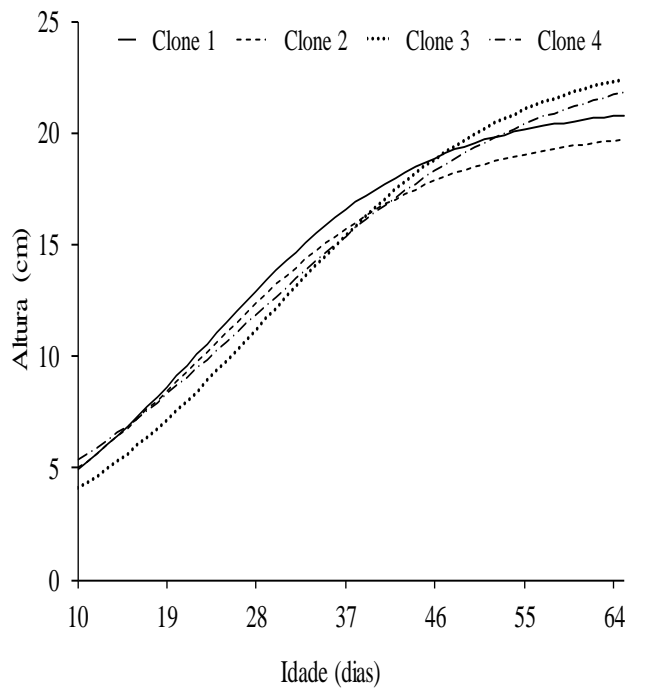

Figura 1. Altura média de mudas clonais de eucalipto ajustadas pelo modelo logístico, em função da idade.

A análise conjunta dos três critérios selecionados para definir a idade de expedição das mudas clonais de eucalipto encontra-se na Figura 2. Com base nas curvas de incremento em altura, e considerando-se a idade técnica de expedição como o momento em que o ICD se iguala ao IMD, verificouse que as mudas dos clones 1 e 2 estariam aptas ao plantio na idade de 37 DAE, enquanto a idade de 46 e 44 dias seria a mais indicada para os clones 3 e 4 , respectivamente. O ICD decresce a partir desse período, devido provavelmente à estagnação de crescimento das mudas, indicando a necessidade de aclimatação das mudas antes de serem expedidas para o campo.

Segundo Mafia et al. (2005), a definição de critérios técnicos de intercepto entre as curvas de incremento corrente diário e incremento médio diário podem ser úteis para a tomada de decisão na avaliação do padrão de qualidade de mudas para expedição. A utilização do intercepto entre curvas de ICD e IMD consolidou-se na área florestal com o objetivo de definir o momento ótimo de corte do povoamento (Campos e Leite 2017), e atualmente vem sendo usada com êxito para determinar variáveis de avaliação da qualidade de mudas florestais, conforme estudos realizados por Melo et al. (2011), Brondani et al. (2012) e Pimentel (2016).

Para os quatro clones analisados, o ajuste do modelo logístico indicou tendência de crescimento em diâmetro ao longo do tempo, com tendência de estabilização em período inferior ao observado para a altura, fato também observado por Trigueiro e Guerrini (2003). Considerando-se como aptas ao plantio as mudas que apresentem diâmetro do colo acima de 2,3 mm, observou-se que esses valores foram atingidos aos $50,57,50$ e 59 DAE para os clones 1, 2, 3 e 4, respectivamente.

Em geral, mudas com maiores diâmetros do colo apresentam-se mais tenras, por possuírem maior acúmulo de reservas na haste, associadas à formação de carboidratos não estruturais, os quais conferem resistência ao tombamento e acamamento das plantas (Kozlowski et al., 2001; Vladan et al. 2016; Singh et al. 2017). Por outro lado, mudas com diâmetros maiores que o recomendado podem apresentar dificuldades de adaptação em campo, conforme observado por Moreira et al. (2016) utilizando mudas clonais de Eucalyptus urophylla $\mathrm{x} E$. grandis mais velhas, as quais apresentavam diâmetros superiores a $3,5 \mathrm{~mm}$. Neste contexto, a utilização da variável diâmetro das mudas deve ser associada a outras variáveis, especialmente a altura, visando uma análise mais rigorosa do momento de expedição das mudas de eucalipto.

Segundo Gomes et al. (2002), as avaliações não destrutivas apresentam uma boa contribuição relativa na análise das mudas, onde o diâmetro e a altura juntos contribuem em $83,19 \%$ na determinação da qualidade.

A relação $\mathrm{H} / \mathrm{DC}$ representa o índice de robustez das mudas, ao associar em um único índice o crescimento das mudas em altura e diâmetro, relacionando o crescimento dos meristemas apicais com os meristemas secundários, responsáveis pela lignificação do caule. Ao analisar a faixa de valores para a relação H/DC considerada ideal por Carneiro (1995), observou-se idades inferiores de expedição das mudas em relação às observadas para as variáveis intercepto ICM e IMD e diâmetro isoladamente. Considerando-se apenas o cenário dos valores da relação H/DC, as mudas teriam idade para expedição a partir de 15 dias, o que resultaria em mudas fisiologicamente imaturas para expedição para o campo.

Apesar da análise da relação H/DC ser importante para a avaliação da qualidade das mudas florestais, uma vez que reflete o acúmulo de reservas, Kratz e Wendling (2016) constataram que a faixa considerada adequada $(5,4$ a 8,1$)$ não é mais indicada para avaliação de mudas clonais de eucalipto, conforme já observado em outros trabalhos, em que a relação H/ DC sempre aparece como superior a oito.

Tais constatações podem ser devido à restrição de volume do recipiente utilizado $\left(55 \mathrm{~cm}^{3}\right)$, possibilitando menores 
acréscimos no diâmetro, ou à utilização de mudas provenientes de material clonal, que pode seguir padrões de crescimento distintos das mudas seminais. Trabalhos realizados com mudas clonais de eucalip to que apresentaram qualidade satisfatória indicam média da relação H/DC entre 8,5 e 11,9 (Toledo et al. 2015; Cerqueira et al. 2017; Dellai et al. 2018; Silva et al. 2018). O presente trabalho corrobora com os demais autores, de forma que é necessária a manutenção das mudas por um período maior no viveiro, para possibilitar a rustificação destas antes da expedição ao campo.

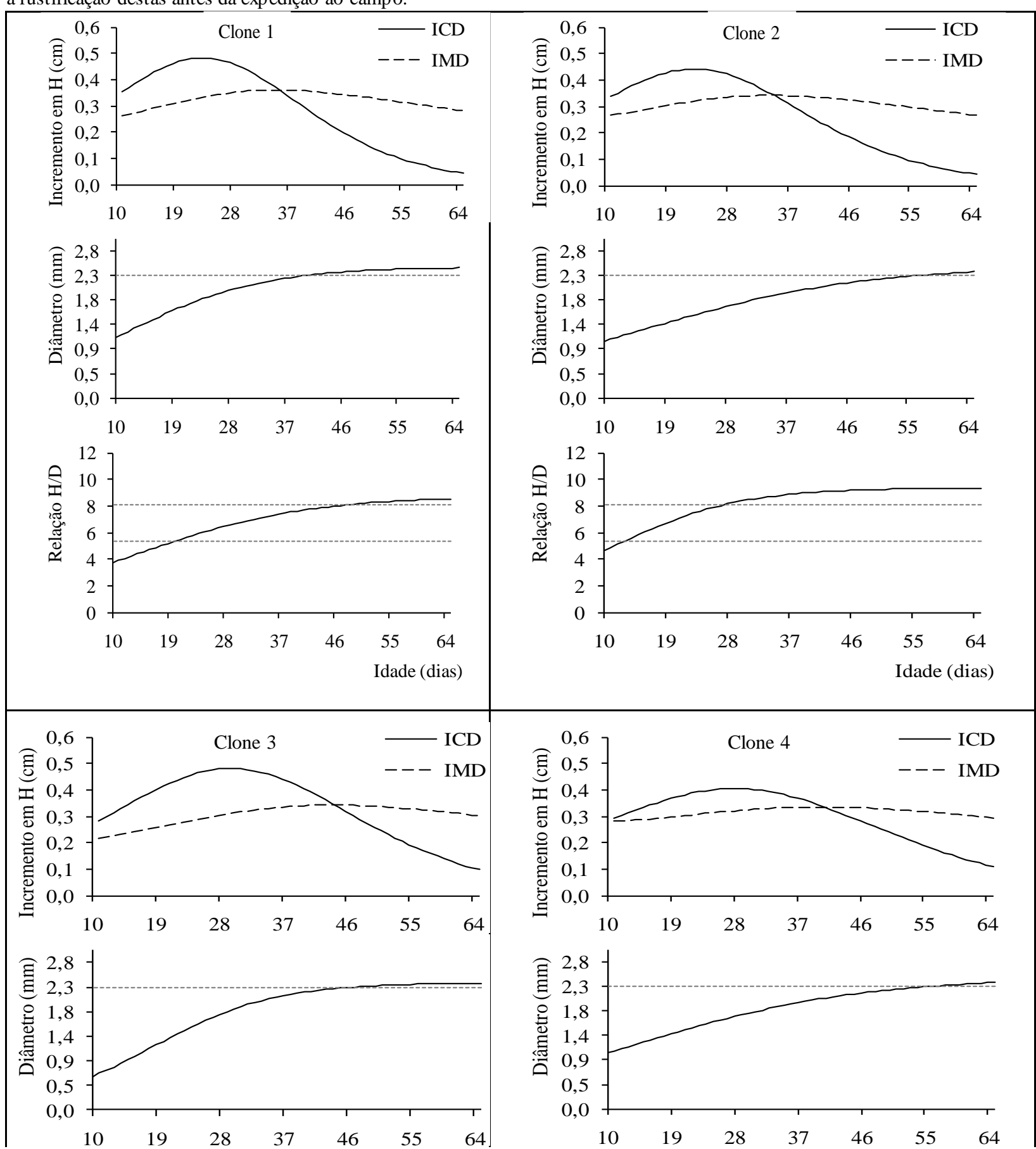

Dessa forma, após análise em conjunto dos critérios intercepto IMD e ICD, diâmetro e relação H/DC, verificou-se que no tempo final de avaliação as mudas atingem ao Critério $_{1}$ (IMD se iguala ao ICD para a variável altura) e ao Critério $2(\mathrm{D} \geq 2,3 \mathrm{~mm}$ ), porém ultrapassa ao intervalo estabelecido no Critério 3 (H/D entre 5,4 e 8,1). Neste contexto as mudas dos clones 1 e 3 estariam aptas ao plantio na idade de 50 DAE, enquanto a idade de 57 e 59 dias seria a mais indicada para os clones 2 e 4 , respectivamente.
Idade (dias) 


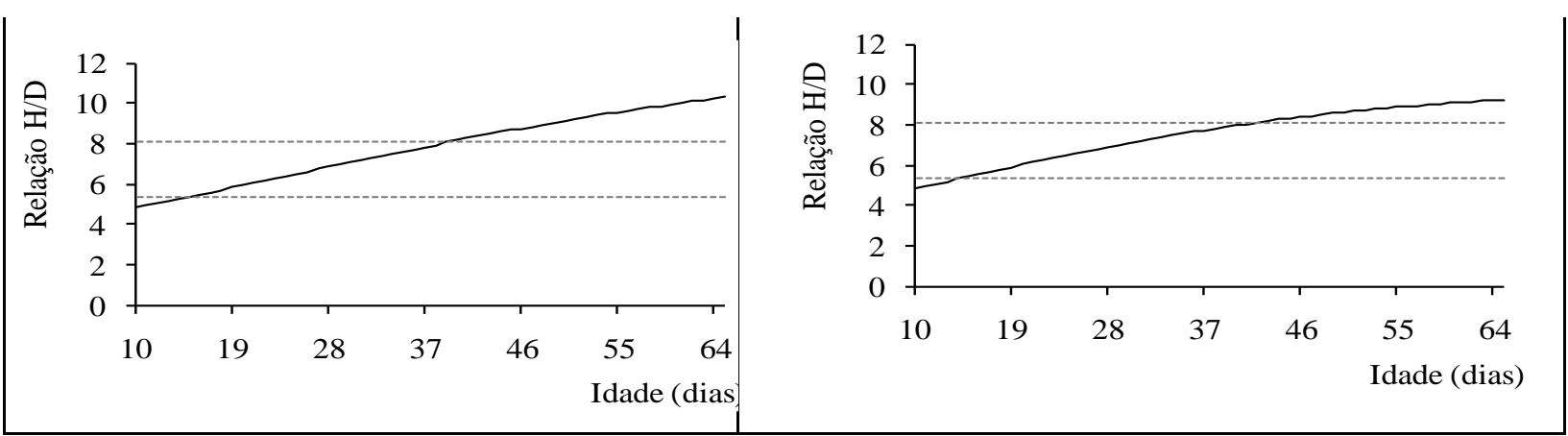

Figura 2. Incremento corrente diário (ICD) e incremento médio diário (IMD) para altura e tendências de crescimento em diâmetro e relação altura/diâmetro $(H / D)$ em função da idade como critério para expedição de mudas de quatro clones de eucalipto. A seta azul representa o momento de expedição

das mudas considerando-se os seguintes critérios estabelecidos neste trabalho: Critério1 (IMD se iguala ao ICD para a variável altura) e Critério2 ( $\mathrm{D} \geq 2,3 \mathrm{~mm})$,

\section{Conclusões}

Conclui-se que a utilização das variáveis altura e diâmetro foi eficiente para estimar a idade de expedição de mudas clonais de eucalipto do viveiro, sendo indicado o uso em conjunto dos seguintes critérios: momento em que o Incremento Médio Diário se iguala ao Incremento Corrente Diário para a variável altura e quando a variável diâmetro é superior a $2,3 \mathrm{~mm}$.

\section{Referências}

Amri E, Lyaruu HVM, Nyomora AS, Kanyeka ZL (2010) Vegetative propagation of African Blackwood (Dalbergia melanoxylon Guill. \& Perr.): effects of age of donor plant, IBA treatment and cutting position on rooting ability. New Forests, 39(2):183-194. doi: 10.1007/s11056-009-9163-6

Ataíde GM, Castro RVO, Santana RC, Dias BAS, Correia ACG, Mendes AFN (2010) Efeito da densidade na bandeja sobre o crescimento de mudas de eucalipto. Revista Trópica, 4(2):21-28.

Brondani GE, Wendling I, Brondani AE, Araújo MA, Silva ALL, Gonçalves AN (2012) Dynamics of adventitious rooting in mini-cuttings of Eucalyptus benthamii $\mathrm{x}$ Eucaly ptus dunnii. Acta Scientiarum 34(2):169-178. doi: 10.4025/actasciagron.v34i2.13059

Campos JCC, Leite HG (2017) Mensuração florestal: perguntas e respostas. $5^{\mathrm{a}}$ ed. Viçosa: Editora UFV. 636p.

Carneiro JGA (1995) Produção e controle de qualidade de mudas florestais. Curitiba: FPR/FUPEF. 451p.

Cerqueira PHA, Azevedo GB, Souza AM, Azevedo GTOS (2017) Adubação residual na produção de mudas clonais de eucalipto. Pesquisa Florestal Brasileira 37(90):119-129. doi: 10.4336/2017.pfb.37.90.934

Costa DBC, Streck NA (2018) Duração da fase de mudas em eucalip to simulada em cenários de aumento de temperatura. $\begin{array}{llll}\text { Ciência Florestal 28(2):1263-1270. doi: } & \end{array}$ $10.5902 / 1980509833378$

Dellai A, Silva RF, Andreazza R (2018) Ectomicorriza no crescimento de Eucalyptus saligna em solo contaminado com cobre. Ciência Florestal 28(2):624-631. doi: $10.5902 / 1980509832052$
Fernandes MM, Sousa FL, Silva JPM, Araújo EF, Fernandes MRM, Nóbrega RSA (2019) Redes Neurais Artificiais na estimação de variáveis biométricas de mudas de espécies florestais produzidas em diferentes substratos. Revista de Ciências Agroveterinárias 18(1):47-58. doi: $10.5965 / 223811711812019047$

Figueiredo FAMMA, Carneiro JGA, Penchel RM, Barroso DG, Daher RF (2011) Efeito das variações biométricas de mudas clonais de eucalipto sobre o crescimento no campo. Revista Árvore 35(1):1-11. doi: 10.1590/S010067622011000100001

Gilbert A, Gray EF, Westoby M, Wright IJ, Falster DS (2016) On the link between functional traits and growth rate: metaanaly sis shows effects change with plant size, as predicted. Journal of Ecology 104(5):1488-1503. doi:10.1111/13652745.12594

Gomes JM, Couto L, Leite HG, Xavier A, Garcia SLR (2002) Parâmetros morfológicos na avaliação da qualidade de mudas de Eucalyptus grandis. Revista Árvore 26(6):655664. doi: 10.1590/S0100-67622002000600002

Gonzaga MIS, Mackowiak C, Almeida AQ, Carvalho Júnior JIT (2018) Sewage sludge derived biochar and its effect on the growth and morphological traits of Eucalyptus grandis W.Hill Ex Maiden seedlings. Ciência Florestal 28(2):687695. doi: 10.5902/1980509832067

Graner EM (2009) Morphophysiological evaluations of the development of pejibaye microplants treated with bioregulators. Dissertation, Escola Superior de Agricultura "Luiz de Queiroz", Universidade de São Paulo. 242p.

Guo YY, Yu HY, Kong DS, Yan F, Zhang YJ. (2016) Effects of drought stress on growth and chlorophyll fluorescence of Lycium ruthenicum Murr. seedlings. Photosynthetica 54(4):524-531.

Hartmann HT, Kester DE, Davies JR FT, Geneve RL (2011) Plant propagation: principles and practices. 8th Edition. São Paulo: Prentice-Hall. 915p.

Indústria Brasileira De Árvores - IBÁ (2018). Relatório anobase 2017.2 Disponível em: <iba.org/images/shared/biblioteca/iba_relatorioanual2017. pdf $>$. Acesso em: 30 jun. 2018.

Mafia RG, Alfenas AC, Siqueira L, Ferreira EM, Leite HG, Cavallazzi JRP (2005) Critério técnico para determinação 
da idade ótima de mudas de eucalipto para plantio. Revista Árvore 29(6):947-953. doi: 10.1590/S010067622005000600014

Melo LA, Xavier A, Paiva HN, Borges SR (2011) Otimização do tempo necessário para o enraizamento de miniestacas de clones híbridos de Eucalyptus grandis. Revista Árvore 35(4): 759-767. doi: 10.1590/S0100-67622011000500001

Melo MR (2017) Influência da bacteriose foliar e a desfolha artificial em mudas de eucalipto no crescimento pósplantio. Dissertação, Faculdade de Ciências Agronômicas, Universidade Estadual Paulista Júlio de Mesquita. 54p.

Moreira GG, Lemos CCZ, Hakamada RE, Silva RML, Pires GT (2014) A qualidade de mudas clonais de Eucalyptus urophylla $\mathrm{x}$ E. grandis impacta o aproveitamento final de mudas, a sobrevivência e o crescimento inicial. Série Técnica IPEF 24(45):33-37.

Pimentel N (2016) Miniestaquia e qualidade de mudas de erva-mate. Dissertação, Centro de Ciências Rurais, Universidade Federal de Santa Maria. 122p.

Pires RN, Bacha AL, Nepomuceno MP, Alves PLCA (2019) Pode o etiltrinexapac estimular o crescimento de mudas de eucalipto? Ciência Florestal 29(1): 385-395. doi: $10.5902 / 1980509815326$

Queiroz DL, Camargo JMM, Dedecek RA, Oliveira EB, Zanol KMR (2018) Absorção e translocação de silício em mudas de Eucalyptus camaldulensis. Ciência Florestal 28(1):632-640. doi: 10.5902/1980509832053

Schumacher MV, Vieira M (2016) Silvicultura do eucalipto no Brasil. Santa Maria: Editora da UFSM, 316p.

Silva FAM, Nunes GM, Zanon A, Guerrini IA, Silva RB (2018) Resíduo agroindustrial e lodo de esgoto como substrato para a produção de mudas de Eucalyptus urograndis. Ciência Florestal 28(2):827-828. doi: $10.5902 / 1980509832101$

Silveira RLVA, Moreira A, Higashi EM (2004) Crescimento e sobrevivência de mudas de eucalipto sob doses de boro cultivadas em condições de viveiro e de campo. Ciência e agrotecnologia 28(2):366-371.

Singh KVA, Husain M, Geelani SN, Ali SR, Parrey AA, Tariq M (2017) Effect of spacing, nitrogen fertilizer (with and without organic manure) and seed bed density on the growth of Allepo pine seedling in nursery. International Journal of Chemical Studies 5(5): 627-634.

Souza-Silva A, Zanetti R (2007) Forrageamento por Atta sexdens rubropilosa Forel, 1908 (Hymenoptera: Formicidae) a campo em mudas de eucalipto pulverizadas ou imersas em soluções de extrato pirolenhoso. Revista Árvore, 31(4):753-759. doi: 10.1590/\$010067622007000400021

STATSOFT, Inc. STATISTICA (data analysis software system), version 10.0. 2017.

Tavanti RFR, Carvalho JB, Troleis MJB, Bellote JLM, Sena KN, Teixeira MCM (2018). Eficiência da adubação boratada no desenvolvimento de mudas de eucalipto Ciência e agrotecnologia 61(4):1-7. doi: 10.22491/rca.2018.2862
Toledo FHSF, Venturin N, Carlos L, Dias BAS, Venturin RP, Macedo RLG (2015) Composto de resíduos da fabricação de papel e celulose na produção de mudas de eucalipto. Revista Brasileira de Engenharia Agrícola e Ambiental 19(7):711-716. doi: 1929/agriambi.v19n7p 711-716

$10.1590 / 1807-$

Trigueiro RM, Guerrini IA (2003) Uso de biossólidos como substratos para produção de mudas de eucalipto. Scientia Florestalis 164(1):150-162.

Vladan I, Jovana D, Zorah M (2016) Initial height and diameter are equally related to survival and growth of hardwood seedlings in first year after field planting. Reforesta 1(2):6-21. doi: 10.21750/REFOR.2.02.17

Xavier A, Wendling I, Silva RL (2013) Silvicultura clonal: princípios e técnicas. Viçosa: Editora UFV. 279p. 